\title{
Neutron-neutron correlation in the halo dissociation of light exotic nuclei
}

\author{
M. T. Yamashita, ${ }^{1}$ T. Frederico, ${ }^{2}$ and Lauro Tomio ${ }^{3}$ \\ ${ }^{1}$ Unidade Diferenciada de Itapeva, Universidade Estadual Paulista, 18409-010 Itapeva, Brasil \\ ${ }^{2}$ Departamento de Física, Instituto Tecnológico de Aeronáutica, Centro Técnico Aeroespacial, 12228-900 São José dos Campos, Brasil \\ ${ }^{3}$ Instituto de Física Teórica, Universidade Estadual Paulista, 01405-900 São Paulo, Brasil
}

(Received 10 March 2005; published 18 July 2005)

\begin{abstract}
We present model results for the two-halo-neutron correlation functions, $C_{n n}$, for the dissociation process of light exotic nuclei modelled as two neutrons and a core. A minimum is predicted for $C_{n n}$ as a function of the relative momentum of the two neutrons, $p_{n n}$, due to the coherence of the neutrons in the halo and final state interaction. Studying the systems ${ }^{14} \mathrm{Be},{ }^{11} \mathrm{Li}$, and ${ }^{6} \mathrm{He}$ within this model, we show that the numerical asymptotic limit, $C_{n n} \rightarrow 1$, occurs only for $p_{n n} \gtrsim 400 \mathrm{MeV} / c$, while such limit is reached for much lower values of $p_{n n}$ in an independent particle model as the one used in the analysis of recent experimental data. Our model is consistent with data once the experimental correlation function is appropriately normalized.
\end{abstract}

DOI: 10.1103/PhysRevC.72.011601 PACS number(s): 25.10.+s, 21.45.+v, 21.10.Gv, 24.10.Cn

The discovery of radioactive exotic weakly bound nuclei, rich in neutrons or protons, beyond the drip line have brought a lot of interest in the nuclear structure and reactions of these unstable nuclei. The traditional nuclear models are unable to describe the long-range correlations between the nucleons of the halo. The large size of the halo has stimulated several experimental and theoretical studies devoted to clarify the new aspects of the structure, stability, and reaction of these nuclei, including astrophysical applications (see, for example, [1-3]).

The effect of the large spatial extension of the neutron halo, was probed in a recent fusion experiment of ${ }^{6} \mathrm{He}$ with ${ }^{238} \mathrm{U}$ target [4]. It was observed a large reaction cross section due to a direct $2 n$ ( $n$ represents a neutron) transfer rather than an enhancement of the complete fusion cross section. In view of the large size of the halo $\left(r_{n n}^{\mathrm{rms}}=5.9 \pm 1.2 \mathrm{fm} \mathrm{[5],} r_{n n}^{\mathrm{rms}}\right.$ is the $n n$ root-mean-square radius) which is comparable to the size of ${ }^{238} \mathrm{U}$ itself, it is most likely that a correlated neutron-neutron $(n n)$ is transferred to ${ }^{238} \mathrm{U}$ while the ${ }^{4} \mathrm{He}$ is still far from the fusion barrier, favoring the $2 n$ transfer process.

A novel view of the complete fusion reaction for large halo nuclei also emerges [6]: It is most probable that the core approaches the fusion barrier of an excited target nucleus that has already absorbed the halo. This interpretation is in line with the concept of an absorptive many-body potential written in the relative coordinates of the core, two neutrons, and the target [7].

The need for a deeper insight into the key aspects of the structure and dynamics of the halo in Borromean three-body systems (where all the subsystems are unbound), like ${ }^{6} \mathrm{He}$ is evident. In this respect, using intensity interferometry applied with a new iterative technique, Marqués et al. [5,8] recently probed the spatial configuration of two-neutron halo systems and estimated the mean-square $n n$ distances as well, considering the dissociation of ${ }^{6} \mathrm{He},{ }^{11} \mathrm{Li}$, and ${ }^{14} \mathrm{Be}$ in the field of a heavy nucleus target. The spatial configuration of ${ }^{11} \mathrm{Li}$ was also studied by Petrascu et al. $[9,10]$. The $n n$ correlation function, $C_{n n}$, is extracted as a function of the relative momentum between the neutrons, $p_{n n}$. As the absolute normalization of the correlation function is an important piece of physical information, one should consider a consistent model for that. We note that, for the asymptotic normalization, in the fit to data, it has been considered a model where the halo neutrons are assumed to be independent [11]. In our understanding, one should assume them as being emitted by a coherence source.

In the present communication we report our results for the $C_{n n}$ of Borromean three-body systems $n-n-A$, where $A$ is the core mass number. We consider ${ }^{6} \mathrm{He},{ }^{11} \mathrm{Li}$, and ${ }^{14} \mathrm{Be}$ within a description of two neutrons and a core forming Borromean systems. We show how to circumvent the major difficulty of the relationship between the initial and final states due to the distorting effects of the reaction: We consider the $n n$ final state interaction (FSI) and the three-body structure of the Borromean system. The $n n$ FSI is shown to play a crucial role in distorting the relative motion of the neutrons and it is a source of interference effects which leads to an unexpected minimum of the $C_{n n}$, as a function of $p_{n n}$, pushing the asymptotic behavior (where $C_{n n}=1$ ) to larger momentum. By comparing our results with the available experimental ones $[5,8,9]$, we found that it is reasonable to expect a different normalization for the data, as will be shown.

We use a three-body model in the limit of zero-range interaction, which retains the essential physics of the weakly bound and large two-neutron halo systems [2,3]. The interaction singularity is tamed in a renormalized zero-range model [12-15] which is appropriate to study weakly bound three-body systems. The model is parametrized by minimal number of physical inputs, which are directly related to known observables: The two-neutron separation energy, $S(2 n)=$ $-E_{n n c}$, the $n n$ and neutron-core $(n-A) s$-wave scattering lengths (or the corresponding virtual or bound state energies).

The neutrons of the halo have a large probability to be found outside the interaction range. Therefore the low-energy properties of these halo neutrons are, to a large extend, model independent as long as few physical input scales are fixed. The model provides a good insight into the three-body structure of halo nuclei, even considering some of its limitations. It is restricted to $s$-wave two-body interactions, with small energies 
for the bound or virtual states. Even in this case, the three-body wave function for the valence neutrons presents configuration mixing due to angular momentum recoupling (see also Refs. [14,16]). We also note that, all the interaction effects, such as higher partial waves in the interaction and/or Pauli blocking effect are, to some extent, included in the model, as long as the experimental two- and three-body energies are supplied. In this perspective, it is of interest to extend the three-body continuum calculation of $C_{n n}$, done in [17] for ${ }^{6} \mathrm{He}$, to analyze the actual data.

The zero-range interaction itself does not have a physical scale, then any calculated observable only depends on the two- and three-body energy scales. The dependence on the three-body scale is a consequence of the Thomas collapse [18] of the three-body system in the limit of a zero-range interaction which demands a three-body scale to supply the system with the physical information of the three-particle short distance configurations. Therefore, any dimensionless observable is a function of the ratios between the input energies, the core mass $A$ and as well as on the nature of the subsystems, bound or virtual [13]. The functional form of each observable written in terms of dimensionless ratios is given by a universal scaling function valid for short-range interactions or large systems [19]. The model is more general and can describe phenomena in atomic and molecular physics such that other large three-body systems with different structures can also be treated and stability and size studies performed (see, e.g., Ref. [3,20]).

We show that the asymptotic limit $C_{n n} \rightarrow 1$ is reached in our model at much higher values of $p_{n n}$ than the ones found in previous data analyses [5,8-10]. Due to coherence of the neutrons in the halo and final state interaction, $C_{n n}$ goes smoothly to the asymptotic limit only after displaying a minimum, as it will be shown.

Particle distributions in the halo were calculated in Refs. $[15,19]$, where the three-body Faddeev equations for the renormalized zero-range two-body interactions were solved. We obtain $C_{n n}$ by using the corresponding three-body wave function with the inclusion of the $n n$ final-state interaction (FSI) [21]. We will show our results in the case of the halo nuclei ${ }^{6} \mathrm{He},{ }^{11} \mathrm{Li}$, and ${ }^{14} \mathrm{Be}$.

For the $n-n-A$ three-body system, $C_{n n}$ is given by

$$
\begin{aligned}
& C_{n n} \equiv C\left(\vec{p}_{n n}\right)=\frac{\int d^{3} q_{n n}\left|\Phi\left(\vec{q}_{n n}, \vec{p}_{n n}\right)\right|^{2}}{\int d^{3} q_{n n} \rho\left(\vec{q}_{n A}\right) \rho\left(\vec{q}_{n^{\prime} A}\right)}, \\
& \vec{q}_{n A} \equiv \vec{p}_{n n}-\frac{\vec{q}_{n n}}{2} \quad \text { and } \quad \vec{q}_{n^{\prime} A} \equiv-\vec{p}_{n n}-\frac{\vec{q}_{n n}}{2},
\end{aligned}
$$

where the one-body density is

$$
\rho\left(\vec{q}_{n A}\right)=\int d^{3} q_{n^{\prime} A}\left|\Phi\left(-\vec{q}_{n A}-\vec{q}_{n^{\prime} A}, \frac{\vec{q}_{n A}-\vec{q}_{n^{\prime} A}}{2}\right)\right|^{2} .
$$

$\Phi \equiv \Phi\left(\vec{q}_{n n}, \vec{p}_{n n}\right)$ is the corresponding breakup amplitude of three-body wave function including the FSI between the neutrons. $\vec{q}_{n n}$ is the relative momentum between the core $A$ and the center of mass of the $n n$ subsystem; and $\vec{p}_{n n}$ the relative momentum between the neutrons.

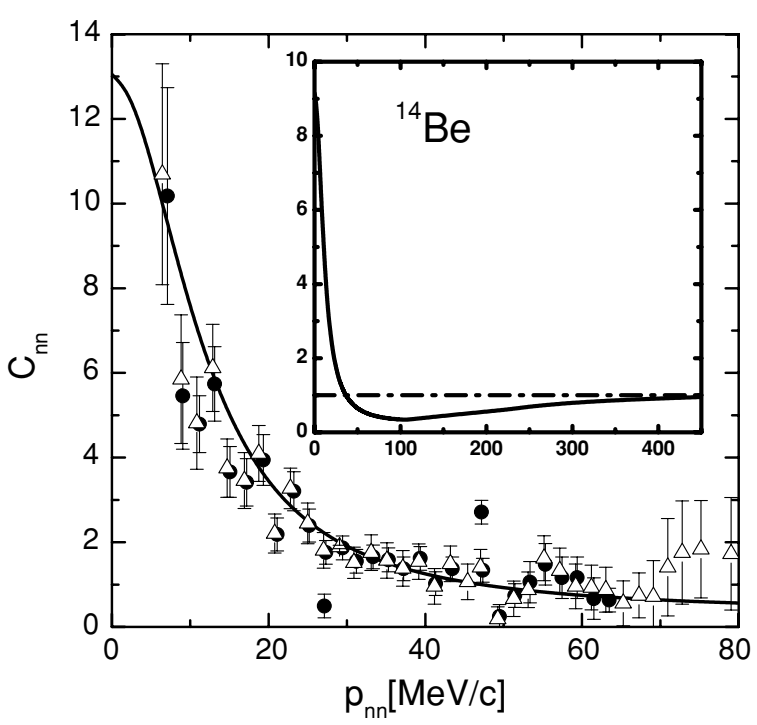

FIG. 1. Two-neutron correlation for the halo of ${ }^{14} \mathrm{Be}$, as a function of the relative $n n$ momentum, $p_{n n}$. The solid curve gives the model results for $S_{2 n}=1.337 \mathrm{MeV}$, and $E_{n A}=0.2 \mathrm{MeV}$. When compared with data, the model result is multiplied by 1.425 . Experimental data are from [5] (open triangles) and [8] (full circles).

The FSI is introduced directly in the inner product $\Phi \equiv\left\langle\vec{q}_{n n} ; \vec{p}_{n n}{ }^{(-)} \mid \Psi\right\rangle$, where the ket $\left|\vec{p}_{n n}{ }^{(-)}\right\rangle$refers to the $n n$ scattered wave given by the Lippmann-Schwinger equation. The correlation function, calculated with the distorted-wave amplitude, assumes a sudden breakup of the halo as the main reaction mechanism. The halo is considered as a coherent source of neutrons, differently from the framework of Ref. [11]. In our picture, the slow halo motion decouples from the fast motion of the core in the field of the target. The distorted wave amplitude $\Phi$ is given by

$$
\Phi=\Psi\left(\vec{q}_{n n}, \vec{p}_{n n}\right)+\frac{1 /\left(2 \pi^{2}\right)}{\sqrt{E_{n n}}-i p_{n n}} \int d^{3} p \frac{\Psi\left(\vec{q}_{n n}, \vec{p}\right)}{p_{n n}^{2}-p^{2}+i \epsilon}
$$

where $\Psi$ is the three-body wave function [15]. $E_{n n}$ is the $n n$ virtual state energy taken as $0.143 \mathrm{MeV}$.

In the framework of Lednicky-Lyuboshits [11], in order to obtain the correlation function $C_{n n}$, the probability density (in configuration space) of the neutron-neutron scattering state is multiplied by the probability density of the relative motion of the halo neutrons in the three-body wave function. Such model for the correlation function was developed from astrophysics, and the possibility to apply it to particle and nuclear physics is valid if one assumes that the particles emitted by the source are independent. We believe this is not the case, when considering the neutrons of the halo of a nuclei. So, in our approach, when the distorted-wave amplitude of Eq. (3) is considered, $|\Phi|^{2}$ contains off-diagonal matrix elements of the two-body densities.

The results of our calculations for $C_{n n}$ of the systems ${ }^{14} \mathrm{Be}$, ${ }^{11} \mathrm{Li}$, and ${ }^{6} \mathrm{He}$ are, respectively, shown in Figs. 1-3. They are shown as functions of $p_{n n}$, and compared with experimental available data $[5,8,9]$. 


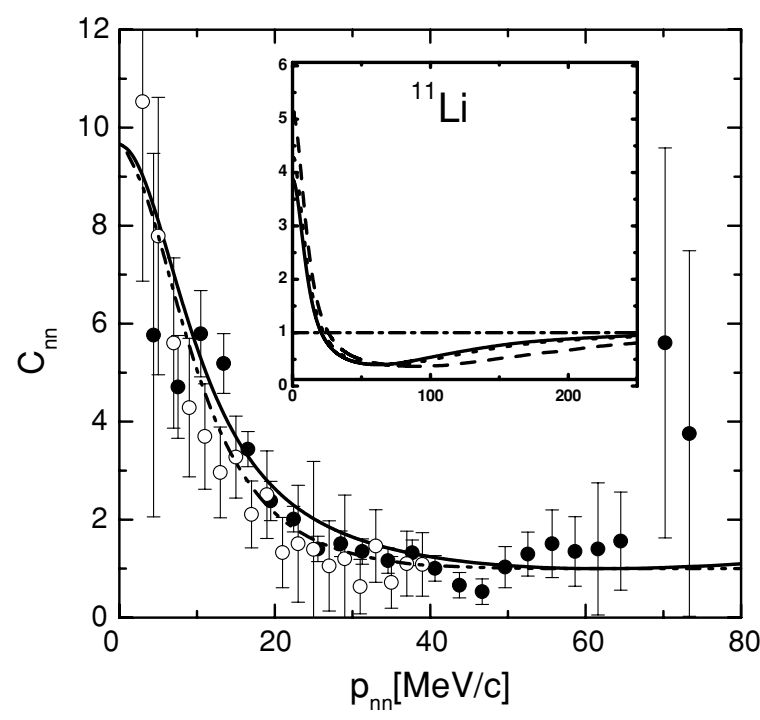

FIG. 2. Two-neutron correlation for the halo of ${ }^{11} \mathrm{Li}$, as a function of the relative $n n$ momentum, $p_{n n}$. The model results (inset) are given for three cases: $S_{2 n}=0.29 \mathrm{MeV}$ and $E_{n A}=0.05 \mathrm{MeV}$ (solid line); $S_{2 n}=0.37 \mathrm{MeV}$ and $E_{n A}=0.8 \mathrm{MeV}$ (dashed line); and, $S_{2 n}=$ $0.37 \mathrm{MeV}$ and $E_{n A}=0.05 \mathrm{MeV}$ (dotted line). In the main body of the figure, the solid curve (when $r_{n n}^{\mathrm{rms}}=8.5 \mathrm{fm}$ [15]) presents the corresponding curve of the inset multiplied by 2.5 ; the dot-dotdashed curve, the model presented in [9] with $r_{n n}^{\mathrm{rms}}=8.3 \mathrm{fm}$. The experimental data are from [5] (full circles) and [9] (empty circles).

In the present Faddeev renormalized zero-range model, as expected, $C_{n n}$ goes asymptotically to unity, as a function of $p_{n n}$. Such limit is shown clearly in all three selected systems that we have analyzed. The results that include the

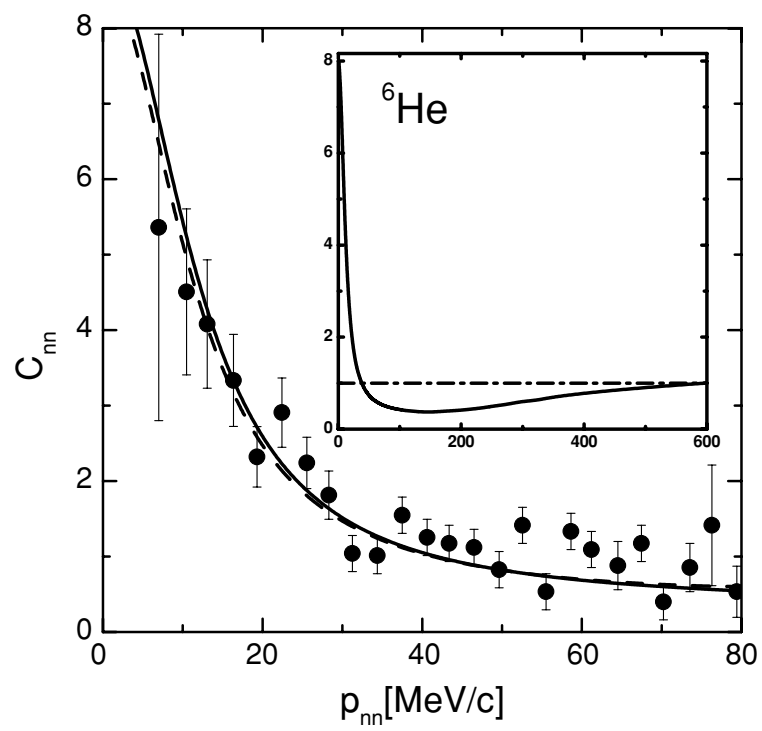

FIG. 3. Two-neutron correlation for the halo of ${ }^{6} \mathrm{He}$, as a function of the relative $n n$ momentum, $p_{n n}$. The solid curve gives the model results for $S_{2 n}=0.973 \mathrm{MeV}$ and $E_{n A}=4 \mathrm{MeV}$; and, the dashed curve, for $S_{2 n}=0.973 \mathrm{MeV}$ and $E_{n A}=0$. When compared with data (extracted from [5]), the model results (in the main body) are conveniently normalized as explained in the text. asymptotic limit are shown in the insets of the figures. As we observe, for example, in the insets of Figs. 1-3, the theoretical $p_{n n}$ asymptotic limits for the systems ${ }^{14} \mathrm{Be},{ }^{11} \mathrm{Li}$, and ${ }^{6} \mathrm{He}$ are, respectively, $\gtrsim 400 \mathrm{MeV} / c, \gtrsim 250 \mathrm{MeV} / c$, and $\gtrsim 500 \mathrm{MeV} / c$. Besides the fact of the qualitative similar behavior, when comparing the results of the insets with the corresponding experimental results, we note a clear scale discrepancy. The striking observation is that the interference effect produced by the inclusion of FSI originates a minimum for $C_{n n} \approx 0.35$, pushing the asymptotic limit to much larger values of $p_{n n}$ than the ones considered in the asymptotic normalization of the experimental data. Our model calls for a different normalization of the experimental $C_{n n}$ obtained in Refs. $[5,8,9]$. We believe that the qualitative picture presented by our model, evidencing a minimum of $C_{n n}$, will survive in a more realistic three-body approach.

In Figs. 1-3, we observe a quite good agreement between theory and data when an appropriate normalization factor is included. Particularly, for ${ }^{14} \mathrm{Be}$, the data are well reproduced by the model with $S_{2 n}=1.337 \mathrm{MeV}$ [22] and with $E_{n A}=$ $0.2 \mathrm{MeV}$ [23] (where $E_{n A}$ is the virtual state energy of $n-{ }^{12} \mathrm{Be}$ ), when our $C_{n n}$ is multiplied by 1.425 , as shown in Fig. 1 (or, $C_{n n} \approx 0.70 C_{n n}^{\exp }$, where $C_{n n}^{\exp }$ is the model fit to experimental data).

We have studied the model sensitivity to the two and three-body binding energies considering the case of ${ }^{11} \mathrm{Li}$. We chose this nucleus because it has the smallest $2 n$ separation energy $S_{2 n}$ among the nuclei we are analyzing. The model results are shown by the three plots presented in the inset of Fig. 2. For a fixed $s$-wave ${ }^{10} \mathrm{Li}$ two-body virtual state energy, $E_{n A}=0.05 \mathrm{MeV}$ [16], we vary $S_{2 n}$ from 0.29 MeV [24] (solid line) to $0.37 \mathrm{MeV}$ [25] (dotted line). They differ only near the origin. Next, to observe the sensitivity to the $s$-wave ${ }^{10} \mathrm{Li}$ two-body virtual state energy, we also plot a curve for $E_{n A}=0.8 \mathrm{MeV}$ [26] and $S_{2 n}=$ $0.37 \mathrm{MeV}$ (dashed line). Increasing the virtual state energy, the "plateau" near the minimum is enlarged and, for $p_{n n}<$ $60 \mathrm{MeV} / c, C_{n n}$ is enhanced and even strongly near zero.

We should note that, as we increase the virtual state energy (reduce the absolute value of $n-{ }^{9} \mathrm{Li}$ scattering length) for a fixed $S_{2 n}$ in ${ }^{11} \mathrm{Li}$, we are shrinking the three-body system (see discussion and Table 1 of Ref. [15]), implying that $C_{n n}$ develops a longer tail in momentum space. In the inset of Fig. 2, this effect is clear and shown by the variation of the $n-{ }^{9} \mathrm{Li}$ virtual state energy from 0.05 to $0.8 \mathrm{MeV}$, while $S_{2 n}$ is kept fixed at $0.37 \mathrm{MeV}$. The increase of $S_{2 n}$ from 0.29 to $0.37 \mathrm{MeV}$, with $E_{n A}$ fixed at $0.05 \mathrm{MeV}$, also implies in reducing the size of the three-body system. This is shown in the inset of Fig. 2 by comparing the solid with dotted lines; the variation is small but consistent with the small variation of $S_{2 n}$. We expect that such typical behavior is general. With respect to the correct asymptotic behavior, one can observe our solid line given in the inset figure. We need to multiply it by a factor 2.5 in order to fit the experimental data, as shown in the main body of the figure. So, in this case we have $C_{n n} \approx 0.40 C_{n n}^{\exp }$. We also compare our calculation with another model, given in [9], which is close to the one presented in [5] in the framework of [11]. We observe that the different behavior between our and other models, essential for the absolute 
normalization of the data, will appear at higher momentum values.

Next, to study the case of ${ }^{6} \mathrm{He}$, it is worthwhile to mention that the $s$-wave $n-{ }^{4} \mathrm{He}$ scattering has a positive scattering length and effective range [27,28], which by extrapolation of the standard effective range expansion produces an unphysical ${ }^{5} \mathrm{He}$ bound state. A similar situation is found in the quartet neutron-deuteron scattering where the large positive scattering length and an effective range produces, by naive extrapolation, a bound state near the threshold. The effective interaction in the case of the $n-d$ system is repulsive and no quartet trinucleon exists, similarly for the $n-{ }^{4} \mathrm{He}$ where the $s$-wave interaction should be effectively repulsive to avoid an unphysical bound state. However, in our model the sign of the $n$-core scattering length is determinant of the occurrence of the bound state.

The experimental $n-{ }^{4} \mathrm{He}$ scattering length is 3.26(3) fm [28], which by naive extrapolation of the effective range in our zero-range model produces an unphysical bound state. Therefore, we arbitrarily made a calculation of $C_{n n}$ with a zero energy $s$-wave ${ }^{5} \mathrm{He}$ and the experimental value of $S_{2 n}$ in ${ }^{6} \mathrm{He}$. Surprisingly, we found a reasonable agreement with the data, as shown in Fig. 3. In this case, our results for $C_{n n}$ need to be multiplied by 1.48 to fit the data, as shown by the dashed line in the main part of the figure $\left(C_{n n} \approx 0.68 C_{n n}^{\mathrm{exp}}\right)$.

Observe that the interaction for the $n-{ }^{4} \mathrm{He}$ should be weaker than the one we are using; and, as the interaction becomes weaker while the three-body energy is kept constant, the three-body system tends to be more compact [15] implying an increase of the typical three-body momentum scale. To mimic this effect, we decrease the attraction of the $n-{ }^{4} \mathrm{He}$ $s$-wave interaction, allowing a virtual $s$-wave state (unphysical), using as a typical magnitude for the scattering length the value of $3 \mathrm{fm}$. Still in this case, as we have multiplied our $C_{n n}$ by 1.12, we obtain a good fit to data, as shown by the solid line of Fig. 3. So, leaving the normalization free, one cannot distinguish between different weakly attractive $s$-wave interactions in the ${ }^{5} \mathrm{He}$, if the $S_{2 n}$ and the $n n$ virtual state energy are fixed.
Alternatively, one may think in using the zero-range $T$-matrix and project out the bound-state, using the experimental value of the $n-{ }^{4} \mathrm{He}$ scattering length. This may effectively weaken the interaction, which could in principle produce a similar effect as we have discussed.

In conclusion, we presented a theoretical study of neutronneutron correlation for Borromean three-body weakly-bound systems, considering three cases where experimental data are available. As the present analysis of halo dissociation in a renormalized zero-range three-body framework contains the main physical scales of weakly bound Borromean systems, we believe that our results for low $n n$ relative energies (below $40 \mathrm{MeV}$ ) are to a large extent universal; i.e., other short-range potential models, with the same low energy scales, will produce similar results. Considering that in our model there are no free parameters, as the inputs are just the physical scales (two- and three-body observables), and $C_{n n}\left(p_{n n}\right) \rightarrow 1$, when $p_{n n} \rightarrow \infty$, one can use it to get the normalization of the data. Our zero-range calculations suggest that the relevant effect of the inclusion of final-state interactions with the halo considered as a coherent source of neutrons is the presence of a minimum in $C_{n n}$ at an intermediate region of $p_{n n}$ (see Figs. 1-3). It will be important to verify this conclusion, as well as possible deviations, within a three-body model with more realistic two-body interactions. Finally, we observe a good agreement between theory and experimental results, provided that the data fits, $C_{n n}^{\exp }$, are appropriately normalized. In view of our findings, it will be very interesting to improve the experiments [5,8-10], particularly at higher momenta, in order to characterize the existence of the minimum in $C_{n n}$.

We would like to thank Professor M. Petrascu for details on results shown in [9] and for a clarifying discussion. For partial support, we also thank Fundação de Amparo à Pesquisa do Estado de São Paulo and Conselho Nacional de Desenvolvimento Científico e Tecnológico.
[1] M. V. Zhukov et al., Phys. Rep. 231, 151 (1993).

[2] E. Nielsen, D. V. Fedorov, A. S. Jensen, and E. Garrido, Phys. Rep. 347, 373 (2001).

[3] A. S. Jensen, K. Riisager, D. V. Fedorov, and E. Garrido, Rev. Mod. Phys. 76, 215 (2004).

[4] R. Raabe et al., Nature (London) 431, 823 (1994).

[5] F. M. Marqués et al., Phys. Lett. B476, 219 (2000).

[6] D. Hinde and M. Dasgupta, Nature (London) 431, 748 (2004).

[7] M. S. Hussein, B. V. Carlson, T. Frederico, and T. Tarutina, Nucl. Phys. 738, 367 (2004).

[8] F. M. Marqués et al., Phys. Rev. C 64, 061301(R) (2001).

[9] M. Petrascu et al., Nucl. Phys. A738, 503 (2004).

[10] M. Petrascu et al., Phys. Rev. C 69, 011602(R) (2004).

[11] R. Lednicky and V. L. Lyuboshits, Sov. J. Nucl. Phys. 35, 770 (1982).

[12] A. E. A. Amorim, L. Tomio, and T. Frederico, Phys. Rev. C 46, 2224 (1992).
[13] S. K. Adhikari, T. Frederico, and I. D. Goldman, Phys. Rev. Lett. 74, 487 (1995); S. K. Adhikari and T. Frederico, ibid. 74, 4572 (1995).

[14] A. E. A. Amorim, T. Frederico, and L. Tomio, Phys. Rev. C 56, 2378(R) (1997).

[15] M. T. Yamashita, L. Tomio, and T. Frederico, Nucl. Phys. A735, 40 (2004).

[16] M. Zinser et al., Phys. Rev. Lett. 75, 1719 (1995); M. Zinser et al., Nucl. Phys. A619, 151 (1997).

[17] B. V. Danilin, T. Rogde, J. S. Vaagen, I. J. Thompson, and M. V. Zhukov, Phys. Rev. C 69, 024609 (2004).

[18] L. H. Thomas, Phys. Rev. 47, 903 (1935).

[19] M. T. Yamashita, R. S. Marques de Carvalho, L. Tomio, and T. Frederico, Phys. Rev. A 68, 012506 (2003).

[20] E. Braaten and H.-W. Hammer, cond-mat/0410417.

[21] D. H. Boal, C.-K. Gelbke, and B. K. Jennings, Rev. Mod. Phys. 62, 553 (1990).

[22] G. Audi and A. H. Wapstra, Nucl. Phys. A595, 409 (1995). 
[23] M. Thoennessen, S. Yokoyama, and P. G. Hansen, Phys. Rev. C 63, 014308 (2000).

[24] I. Tanihata, J. Phys. G 22, 157 (1996).

[25] D. Lunney, private communication; the value $S_{2 n}=0.37 \mathrm{MeV}$ is above the upper bound 0.32 of [24].
[26] K. H. Wilcox et al., Phys. Lett. B59 142 (1975).

[27] R. A. Arndt, D. D. Long, and L. D. Roper, Nucl. Phys. A209, 429 (1973).

[28] Data from Neutron News, Vol. 3, No. 3, 1992, pp. 29-37; http://www.ncnr.nist.gov/resources/n-lengths/ 